\title{
ON THE COEFFICIENTS OF CUSP FORMS
}

\author{
DINAKAR RAMAKRISHNAN \\ To Robert Langlands
}

\section{Introduction}

Let $F$ be a number field, and let $\pi$ be a cuspidal, unitary automorphic representation of $G L\left(n, \mathbb{A}_{F}\right)$. For almost all finite places $v$, let $A_{v}(\pi)$ denote the associated (Langlands) conjugacy class in $G L(n, \mathbb{C})$, which is represented by a diagonal matrix $\left[\alpha_{1, v}, \ldots, \alpha_{n, v}\right]$. The trace of this class is denoted $a_{v}$. The general Ramanujan (or purity) conjecture predicts that each $\alpha_{j, v}$ has absolute value 1. This is clearly true for $n=1$, and a series of deep theorems asserts that it holds for $(n=2, F$ totally real) if $\pi$ corresponds to a holomorphic eigenform ([D], $[\mathrm{DS}],[\mathrm{BL}],[\mathrm{C}],[\mathrm{W}],[\mathrm{T} 1],[\mathrm{BR}])$. On the other hand, one knows unconditionally that $\left|\alpha_{j, v}\right|$ is bounded by $(N v)^{1 / 2-1 /\left(n^{2}+1\right)}$ for any $n$ ([LRS]), in fact (strictly) by $(N v)^{1 / 5}$ for $n=2$ and any $F$ ([Sh 1,2$\left.]\right)$. For $G L(2) / \mathbb{Q}$, one has the still stronger bound $\left|\alpha_{j, v}\right| \leq(N v)^{5 / 28}$ ([BDHI]; see also [LRS]). If the analyticity of (twists of) the symmetric fourth power $L$-functions of $G L(2)$ is estabished, and some progress has been made in this direction by D. Ginzburg, then one would be able to replace $5 / 28$ by $1 / 6$. Striking and deep though these results are, we are still far from the conjecture.

In this paper we certainly do not prove the conjecture, but we try to approach the problem from another direction. Our object is to understand better, for $G L(2)$, the structure of the set of primes $v$ in $F$ where the conjecture does hold. For $n \geq 3$, we still get information on the trace $a_{v}$, but this is weaker than knowing the conjecture. For any set $X$ of primes, denote by $\underline{\delta}(X)(\operatorname{resp} . \bar{\delta}(X))$ the lower (resp. upper) Dirichlet density (see below) of $X$, so that $\underline{\delta}(X) \leq \bar{\delta}(X)$, with equality holding iff $X$ has a density. Our main result is

Theorem A. For every cuspidal, unitary automorphic representation $\pi$ of $G L\left(n, \mathbb{A}_{F}\right)$, let $S(\pi)$ denote the set of primes where $\left|a_{v}\right| \leq n$. If $n=2$, we have

$$
\underline{\delta}(S(\pi)) \geq 9 / 10 \text {. }
$$

If $n \geq 3$, then $\underline{\delta}(S(\pi)) \geq 1-1 / n^{2}$.

One sees easily that, for $G L(2), S(\pi)$ is precisely the set of primes where the Ramanujan conjecture holds (cf. Remark 4.10).

Received October 28,1996.

Supported by National Science Foundation grant DMS-9501151. 
Note that our theorem applies in particular to any (cuspidal) Maass form $f$ on the upper half plane $\mathcal{H}$ relative to a congruence subgroup $\Gamma \subset \operatorname{SL}(2, Z)$, which is an eigenfunction for Hecke operators, and to eigenforms over arbitrary $F$ which are conjecturally associated to elliptic curves over $F$.

Using base change $([\mathrm{AC}])$, one can in fact establish the following refinement of our result: Let $E / F$ be a finite solvable Galois extension with Galois group $G$, and let $X$ be the set of primes (in $F$ ) which split completely in $E$. Then $\underline{\delta}(S(\pi) \cap X)$ is greater than or equal to $9 / 10|G|$ (resp. $\left.\left(n^{2}-1\right) / n^{2}|G|\right)$ for $G L(2)$ (resp. $G L(n), n \geq 3$ ).

The surprise for us was not that the conjecture is provable for a positive proportion of primes (for $G L(2)$ ), but that it can be shown to hold for such a high proportion. The proof is given in section 4 after some preliminaries. It uses some known results on the Rankin-Selberg and symmetric power $L$-functions, along with the properties of isobaric sums of automorphic representations, and the key step involves a careful study of the behavior near $s=1$ of suitable incomplete Euler products over infinite sets of primes. A consequence of the method is that, each time there is progress in understanding some symmetric $m$ th power $L$-function, then the density of $S(\pi)$ will rise accordingly, approaching 1 for $m$ large.

Our method in fact gives, for any fixed $t$, some information on when the upper (resp. lower) density of the set $S(\pi, t)=\left\{v|| a_{v} \mid \leq t\right\}$ is strictly less than 1 (resp. greater than 0$)$. This leads to the following

Theorem B. Let $\pi$ be a unitary, cuspidal automorphic representation of $G L\left(n, \mathbb{A}_{F}\right)$. Assume that for all but a finite number of $v$, the numbers $\alpha_{j, v}$ lie in a fixed disk in $\mathbb{C}$. Fix an $\epsilon>0$.

(a) If $n=2$, there is a set $S$ of primes of positive lower density such that

$$
\left|a_{v}\right|>\sqrt{2}-\epsilon, \quad \forall v \in S .
$$

(b) Let $n$ be arbitrary, and $\pi$ self-dual. Then, for every $r \in \mathbb{Q}$, there are sets $S_{j}=S_{j}(r), j=1,2$, of primes of positive lower density such that

$$
a_{v} \in\left(-\left(r+\sqrt{r^{2}+4}\right) / 2-\epsilon,\left(-r+\sqrt{r^{2}+4}\right) / 2+\epsilon\right), \quad \forall v \in S_{1} ;
$$

and

$$
a_{v} \notin\left[-\left(r+\sqrt{r^{2}+4}\right) / 2+\epsilon,\left(-r+\sqrt{r^{2}+4}\right) / 2-\epsilon\right], \quad \forall v \in S_{2} .
$$

This is consistent with the general Sato-Tate conjecture ([L2], [Se]). For $G L(2)$, it provides a mild complement to an elegant result of Serre ([Sh2], appendix), and it has intersection with some work of M.R.Murty $([\mathrm{Mu}])$. To elaborate, take $n=2, F=\mathbb{Q}$, and $\pi$ cuspidal of trivial central character with $\pi_{\infty}$ in the discrete series, i.e., defined by a holomorphic newform $f$ (on the upper half plane $\mathcal{H}$ ) of weight $2 k \geq 2$, level $N \geq 1$, and trivial character. If $\left\{c_{p}\right\}$ denotes the system of Hecke eigenvalues of $f$, then $a_{p}=c_{p} p^{-(2 k-1) / 2}$, and thanks to Deligne $([\mathrm{D}])$, we may write $a_{p}$, for every $p$ not dividing $N$, as $2 \cos \theta_{p} \in[-2,2]$, 
for some $\theta_{p} \in[0, \pi]$. Suppose $f$ is not monomial, i.e., not defined by a Hecke character of a quadratic extension of $\mathbb{Q}$. Then the Sato-Tate conjecture asserts that the angles $\theta_{p}$ are uniformly distributed relative to the measure $\frac{2}{\pi} \sin ^{2} \theta d \theta$. A consequence of this conjecture is that given any angular sector there is a set $S$ of primes of positive density such that $\theta_{p}$ lies in this sector for all $p$ in $S$. Serre's theorem asserts that, for every $\epsilon>0$, there exist sets $S^{+}$and $S^{-}$ of primes of positive lower density such that $a_{p}$ is greater (resp. less) than $2 \cos (2 \pi / 7)-\epsilon$ (resp. $-2 \cos (2 \pi / 7)+\epsilon)$, for all $p$ in $S^{+}$(resp. $S^{-}$). (Note that $2 \cos (2 \pi / 7)=1.24697961 \ldots<\sqrt{2}=2 \cos (\pi / 4)=1.414 \ldots)$ If the symmetric fifth power $L$-function of $\pi$ is invertible at $s=1$, then either Serre's method or ours will lead to the same result (see Prop. 5.6), i.e., that there are sets $S^{+}$and $S^{-}$of primes of positive lower density such that $a_{p}$ is greater (resp. less) than $\sqrt{2}-\epsilon($ resp. $-\sqrt{2}+\epsilon)$, for all $p$ in $S^{+}\left(\right.$resp. $\left.S^{-}\right)$.

The referee has pointed out that for such a $\pi$, i.e., one defined by a nonmonomial holomorphic newform $f$ with trivial character, the assertion of part (a) (of Theorem B above) is already found in [Mu (Corollary 2 to Theorem 4). But it should perhaps be noted that, while Murty's aproach does yield such a result in this case, the write-up there is not quite complete as it seemingly relies on knowing the holomorphy in $\Re(s) \geq 1 / 2$ of the symmetric power $L$-functions of $\pi$, for all $n \leq 4$, which is well believed but unknown. However, a careful perusal of Murty's argument shows that the only time this holomorphy is used there is in establishing the non-vanishing of these $L$-functions on the line $s=1$, which one does know to be true in a different way (see [Sh2]). It should be noted that the arguments in $[\mathrm{Mu}]$ also give good $\Omega$-type results.

Given any unitary, cuspidal automorphic representation $\pi$ of $G L\left(2, \mathbb{A}_{F}\right)$, one can consider the infinite family of symmetric power $L$-functions $L\left(s, \pi, \operatorname{sym}^{n}\right)$, $n \geq 0$, whose Euler factors at unramified primes $v$ are given by

$$
L_{v}\left(s, \pi, \operatorname{sym}^{n}\right)=\left(1-\operatorname{sym}^{n}\left(A_{v}(\pi)\right)(N v)^{-s}\right)^{-1} .
$$

As it was pointed out by Langlands in 1967 ([L1]), the Ramanujan conjecture for $\pi$ is equivalent to the absolute convergence of each (and every) $L\left(s, \pi, s^{n} m^{n}\right.$ ) in $\Re(s)>1$.

Theorem C. Let $\pi$ be a unitary, cuspidal automorphic representation of $G L\left(2, \mathbb{A}_{F}\right)$. Fix any $\epsilon>0$, and an integer $n \geq 1$. Then there exists a set $S=S(\epsilon, \pi, n)$ of primes of density 1 such that the incomplete Euler product $L_{S}\left(s, \pi\right.$, sym $\left.^{n}\right)$ converges in $\{\Re(s)>1+\epsilon\}$.

All our results in this paper remain valid over function fields (in one variable) over a finite field. But one knows quite a bit for function fields due to the works of Drinfeld, Kazhdan, Flicker, Laumon and Lafforgue (see [Lf]); the general Ramanujan conjecture is known to be true if $n$ is 2 or odd, for example. It should also be remarked that for special classes of (essentially) self-dual representations $\pi$ of $G L(n) / \mathbb{Q}([\mathrm{C}])$ or $G L(2) / \mathbb{Q}(\sqrt{D}), D<0([\mathrm{~T}])$, with regular infinity type, the Ramanujan bound is known. But all these representations, 
just like the holomorphic modular forms over totally real fields, are understood via arithmetical geometry, directly or by cleverly using (in addition) functoriality and/or congruences, and they ultimately rely on Deligne's proof of the Weil conjectures. The main aim of this paper is to make (modest) progress on those $\pi$ for which no geometric recourse appears possible.

In sum, our method is to fix a cusp form $\pi$ on $G L(n) / F$, and analyze the set $S(\pi)$ of $v$ where the Ramanujan conjecture holds. We believe that to know that $S(\pi)$ is not small is useful even beyond acquiring evidence for the conjecture. To give an example, combining Theorem A with a mild extension (replacing density by lower density) of the refined strong multiplicity one theorem ([Ra]), one can show that if the local comonents of two cusp forms $\pi, \pi^{\prime}$ on $G L(2) / F$ are isomorphic at almost all places in $S(\pi) \cap S\left(\pi^{\prime}\right)$, then $\pi$ and $\pi^{\prime}$ are globally isomorphic. An alternate problem would be to fix a place $v$ and study the set $S(v)$ of cusp forms which satisfy the conjecture at $v$, and this is what is done in $[\mathrm{Sa}]$ for the case $(n=2, F=\mathbb{Q})$.

\section{Two basic lemmas}

First let us recall the basic notions of density for sets of primes. We will fix a number field $F$ in what follows, and let $P$ denote a prime ideal in the ring of integers $\mathcal{O}_{F}$ with norm $N(P)$.

Definition 1.1. Let $S$ be a set of primes in $F$. Then the upper (resp. lower) Dirichlet density of $S$ is given by

$$
\bar{\delta}(S)=\varlimsup_{s \rightarrow 1^{+}}-\frac{\sum_{P \in S} N(P)^{-s}}{\log (s-1)}, \quad\left(\operatorname{resp} . \underline{\delta}(S)=\underline{\lim }_{s \rightarrow 1^{+}}-\frac{\sum_{P \in S} N(P)^{-s}}{\log (s-1)}\right) .
$$

One says that $S$ has a (Dirichlet) density, denoted $\delta(S)$, when the upper and lower densities are equal. For example, when $S$ is the set of all but a finite number of primes, it has a density with $\delta(S)=1$. For general $S$, one knows that

$$
0 \leq \underline{\delta}(S) \leq \bar{\delta}(S) \leq 1 .
$$

It is helpful to note that, if $X=S \cup T$ is a (disjoint) partition of a set of primes, then one has

$$
\underline{\delta}(S) \geq \underline{\delta}(X)-\bar{\delta}(T) .
$$

Let $\mathcal{D}^{*}$ denote the set of all Dirichlet series $L(s)$, absolutely convergent in $\Re(s)>1$ with an Euler product

$$
L(s)=\prod_{P} L_{P}(s)
$$

where

$$
L_{P}(s)=1+\sum_{m=1}^{\infty} a\left(P^{m}\right) N(P)^{-m s} .
$$


Here $P$ runs over all the (finite) primes in $F$. Observe that $\mathcal{D}^{*}$ is a group. Indeed, the group operation is just multiplication of Euler products (as above), and the unit element is simply 1 (with $a\left(P^{m}\right)=0$ for all $\mathrm{P}$ and $m>0$ ). More importantly, the inverse of any $L(s)$ is none other than $\prod_{P} L_{P}(s)^{-1}$, which is in $\mathcal{D}^{*}$ since each of the local factors $L_{P}(s)$ has 1 as the constant term and is hence invertible, and the global product analytic and non-zero in $\{\Re(s)>1\}$.

Let $L(s)$ be in $\mathcal{D}^{*}$. Then for every set $S$ of primes (in $F$ ), we associate an incomplete Euler product by

$$
L_{S}(s)=\prod_{P \in S} L_{P}(s)
$$

We will denote by $L^{S}(s)$ the Euler product $\prod_{P \notin S} L_{P}(s)$. Clearly, we may view $L_{S}(s)$ and $L^{S}(s)$ as elements of $\mathcal{D}^{*}$.

Let log denote the single valued branch of the logarithm in $\Re(s)>0$, normalized to be real valued on the positive real line.

We will now introduce the following two hypotheses, the first of which will (turn out to) be satisfied by all the $L$-functions we will study (in the ensuing sections), and the second by most.

(H1) The function $\lambda(L, s):=s \rightarrow-(\log (s-1))^{-1} \log L(s)$, which is well defined in $\Re(s)>1$, has finite upper and lower limits, denoted $\bar{\lambda}(L)$ and $\underline{\lambda}(L)$ respectively, as s approaches 1 from the right on the real line.

(H2) $\bar{\lambda}(L)=\varlimsup_{s \rightarrow 1^{+}}(\log (s-1))^{-1} \sum_{P} a(P) N(P)^{-s}$, and similarly for $\underline{\lambda}(L)$, with $\varlimsup$ lim replaced by $\underline{\text { lim. }}$.

These two hypotheses are satisfied, for example, by the incomplete Dedekind Zeta function relative to a set $S$ of primes, namely

$$
\zeta_{F, S}(s)=\prod_{P \in S}\left(1-N(P)^{-s}\right)^{-1}=\prod_{P \in S}\left(\sum_{m \geq 0} N(P)^{-m s}\right) .
$$

In this case, $\log \zeta_{F, S}(s)=\sum_{P \in S} \sum_{m \geq 1} \frac{1}{m} N(P)^{-m s}$ (in $\Re(s)>1$ ), and $\bar{\lambda}\left(\zeta_{F, S}\right)$ (resp. $\underline{\lambda}\left(\zeta_{F, S}\right)$ ) equals $\bar{\delta}(S)$ (resp. $\underline{\delta}(S)$ ).

Note also that the set of $L(s)$ in $\mathcal{D}^{*}$ satisfying either of the hypotheses is a subgroup.

Lemma 1.3. Let $L(s) \in \mathcal{D}^{*}$ satisfy the hypotheses (H1) and (H2). Assume further that the coefficients $\{a(P)\}$ are real and are bounded in absolute value. Fix an $\epsilon>0$. Then there exists sets $S, T$ of primes such that

$$
a(P)<\bar{\lambda}(L)+\epsilon, \quad \forall P \in S, \quad \text { and } \quad a(P)>\underline{\lambda}(L)-\epsilon, \quad \forall P \in T .
$$

We can do much better (in one direction) when $L(s)$ satisfies the following positivity hypothesis: 
(P) $\log L(s)$ defines (in $\Re(s)>1$ ) a Dirichlet series with real, non-negative coeficients.

Lemma 1.5. Let $L(s) \in \mathcal{D}^{*}$ satisfy the hypotheses $(\mathrm{H} 1)$ and $(\mathrm{P})$. Fix a positive real number $t$. Then we have

$$
\bar{\delta}(\{P \mid a(P) \geq t\}) \leq \frac{\bar{\lambda}(L)}{t} .
$$

In particular, if $t>\bar{\lambda}(L)$, then the set

$$
S(t):=\{P \mid a(P) \leq t\}
$$

has positive lower density.

It is important to note that in this lemma, we are not assuming that the coefficients are bounded, and the hypothesis (H2) is not needed.

Proof of Lemmas 1.3 and 1.5. Let $L(s) \in \mathcal{D}^{*}$ satisfy (H1) and (H2) and have real, uniformly bounded coeficients. Let $t$ be a real number, and suppose that $a(P) \geq t$, for all $P$ in a set $X$ of primes of upper density 1 . Then we obtain (using (H1) and (H2)) the following:

$$
\bar{\lambda}(L) \geq \varlimsup_{s \rightarrow 1^{+}} \frac{-\sum_{\text {all } P} a(P) N(P)^{-s}}{\log (s-1)} \geq \varlimsup_{s \rightarrow 1^{+}} \frac{-t \sum_{P \in X} N(P)^{-s}}{\log (s-1)}=t .
$$

Note that the middle step is justified as $X$ has (upper) density 1 . Indeed, since all the $a(P)$ are bounded in absolute value by some $R>0$, we have the following, with $S$ denoting the complement of $X$ :

$$
\varlimsup_{s \rightarrow 1^{+}}\left|\Re\left(\frac{-\sum_{P \in S} a(P) N(P)^{-s}}{\log (s-1)}\right)\right| \leq \varlimsup_{s \rightarrow 1^{+}} \frac{R \sum_{P \in S} N(P)^{-s}}{-\log (s-1)}=0 .
$$

Similarly, the contribution from the imaginary part of the sum over $S$ also vanishes.

Thus if $t>\bar{\lambda}(L)$, the set $X$ must have lower density $<1$; hence $S$ has upper density $>0$. Taking $t=\bar{\lambda}+\epsilon$, we get the first assertion of Lemma 1.3.

Suppose $a(P) \leq t$, for some $t$, for all $P$ in a set $Y$ of lower density 1 . Then, arguing as above, taking $\underline{\lim }$ instead of $\varlimsup$ lim, we see that $\underline{\lambda}(L) \leq t$. So if $t=$ $\underline{\lambda}(L)-\epsilon, Y$ cannot have lower density 1 ; hence its complement, say $T$, has positive upper density. This finishes the proof of Lemma 1.3.

Now assume that $L(s)$ satisfies $(\mathrm{H} 1)$ and $(\mathrm{P})$, but without the hypothesis that the coefficients are bounded. (We also do not assume that $\pi$ satisfies (H2).) For any real number $t$, let $X=X(t)$ denote (as above) the set of primes $P$ where $a(P) \geq t$. Then we have (by positivity)

$$
\bar{\lambda}(L) \geq \varlimsup_{\lim _{s \rightarrow 1^{+}}} \frac{-\sum_{\text {all } P} a(P) N(P)^{-s}}{\log (s-1)} \geq \varlimsup_{\lim _{s \rightarrow 1^{+}}} \frac{-t \sum_{P \in X} N(P)^{-s}}{\log (s-1)}=t \bar{\delta}(X) .
$$

Here the middle step is justified as the coefficients $a(P)$ are non-negative. Thus we get $\bar{\delta}(X) \leq \frac{\bar{\lambda}(L)}{t}$, as asserted. 


\section{Proof of Theorem C}

First we will establish a general result for $G L(n)$, and then specialize to $G L(2)$ to get what we want. Recall that given cuspidal automorphic representations $\pi_{1}, \ldots, \pi_{r}$ of $G L\left(n_{1}, \mathbb{A}_{F}\right), \ldots, G L\left(n_{r}, \mathbb{A}_{F}\right)$, for some positive integers $n_{1}, \ldots n_{r}$, one can form their isobaric sum ([L], [JS], [HR]) $\pi:=\pi_{1} \boxplus \ldots \boxplus \pi_{r}$, which is an automorphic representation of $G L\left(n, \mathbb{A}_{F}\right)$, with $n=n_{1}+\ldots+n_{r}$, such that at every unramified place $v$, one has

$$
a_{v}(\pi)=a_{v}\left(\pi_{1}\right)+\ldots+a_{v}\left(\pi_{r}\right) .
$$

Lemma 3.1. Let $\pi$ be a unitary, isobaric automorphic representation of $G L\left(n, \mathbb{A}_{F}\right)$ with coefficients $a(P)=a_{P}(\pi)$. Fix $t>0$. Then the set of primes $P$ such that $|a(P)| \geq N(P)^{t}$ has density zero.

Proof. Put

$$
L(s)=L(s, \pi \times \bar{\pi}),
$$

where the function on the right is the Rankin-Selberg $L$-function attached to the pair $(\pi, \bar{\pi})([\mathrm{JPSS}],[\mathrm{Sh} 1])$. One knows (see [HR]) that $\log L(s)$ is of positive type, i.e., it has non-negative coefficients as a Dirichlet series. One gets the following inequality for any set $S$ of primes (for all real $s>1$ )

$$
\log L(s) \geq \sum_{P \in S}|a(P)|^{2} N(P)^{-s} .
$$

Now suppose $S$ has upper density $\alpha>0$, and that, for some $t>0, a(P) \geq N(P)^{t}$ for all $P$ in $S$. Then we obtain, from (3.2),

$$
\log L(s) \geq \sum_{P \in S} N(P)^{2 t-s} .
$$

Since $L(s)$ has an Euler product and is hence invertible in $\{\Re(s)>1\}$, the left hand side of (3.3) remains finite as one takes the lim sup as $s$ goes to $1+2 t$. But the right hand side approaches $\alpha \log (1 /(s-1))$, a contradiction $($ as $\alpha>0)$.

Now we show how this lemma implies Theorem C. Indeed, let $\pi$ be a unitary, cuspidal automorphic representation of $G L\left(2, \mathbb{A}_{F}\right)$ of central character $\omega$ and unramified conjugacy classes $A_{v}(\pi)=\left[\alpha_{v}, \beta_{v}\right]$, so that $a_{v}(\pi)=\alpha_{v}+\beta_{v}$ and $\omega_{v}\left(\varpi_{v}\right)=\alpha_{v} \beta_{v}$, where $\varpi_{v}$ is a uniformizer at $v$. For any $n \geq 1$, we have

$$
A_{v}\left(\pi, \operatorname{sym}^{n}\right)=\left[\alpha_{v}^{2 j-n} \omega_{v}\left(\varpi_{v}\right)^{n-j}\right]_{j=0}^{n} .
$$

Consequently, if $X$ denotes the set of places which are archimedean or ramified (for $\pi$ ), we have (in $\Re(s)>1$ )

$$
\log L^{X}\left(s, \pi, \operatorname{sym}^{n}\right)=\sum_{m \geq 1} \sum_{v \notin X} a_{v^{m}}\left(\pi, \operatorname{sym}^{n}\right) m^{-1}(N v)^{-m s},
$$


where, for every $v \notin X$ and $m \geq 1$,

$$
a_{v^{m}}\left(\pi, \operatorname{sym}^{n}\right)=\sum_{j=0}^{n} \alpha_{v}^{m(2 j-n)} \omega_{v}\left(\varpi_{v}\right)^{m(n-j)} .
$$

Since $\omega$ is unitary, the assertion (of Theorem C) will then follow if we show that, for any $t>0$, we can find a set $S$ of density 1 such that

$$
(N v)^{-t / n} \leq\left|\alpha_{v}\right| \leq(N v)^{t / n} \text {. }
$$

This bound on $\alpha_{v}$ is implied by the bound $\left|a_{v}(\pi)\right| \leq 2(N v)^{t / n}$. But the set $T$ of finite places $v$ where $\left|a_{v}(\pi)\right| \geq 2(N v)^{t / n}$ has density zero. So we are done by choosing $S$ to be the complement of $T$.

\section{Proof of Theorem A}

Let $\eta$ be an arbitrary unitary, isobaric automorphic representation of $G L\left(n, \mathbb{A}_{F}\right)$. Set

$$
L(s)=L(s, \eta \times \bar{\eta}) .
$$

We have seen that $L(s)$ satisfies $(\mathrm{H} 1)$ and $(\mathrm{P})$. Suppose

$$
\eta=\boxplus_{j=1}^{r} m_{j} \pi_{j},
$$

where each $\pi_{j}$ is cuspidal. Then one knows (cf. [JS]) that

$$
\bar{\lambda}(L)=\lambda(L)=\sum_{j=1}^{r} m_{j}^{2} .
$$

Put

$$
T(\eta, t)=\left\{v|| a_{v}(\eta) \mid \geq t\right\} .
$$

Then, since $a_{v}(\eta \times \bar{\eta})=\left|a_{v}(\eta)\right|^{2}$, we have (by Lemma 1.5)

$$
\bar{\delta}(T(\eta, t)) \leq \frac{1}{t^{2}} \sum_{j=1}^{r} m_{j}^{2} .
$$

First let $\eta$ be cuspidal, and take $t=n$. Then we get $\bar{\delta}(T(\eta, n)) \leq 1 / n^{2}$. Thus the complement of $T(\eta, n)$ has lower density greater than or equal to $1-1 / n^{2}$. This proves the assertion of Theorem A for $n \geq 3$.

Now let $\pi$ be a (unitary) cuspidal automorphic representation of $G L\left(2, \mathbb{A}_{F}\right)$ with coefficients $a_{v}$ and central character $\omega$. By a theorem of Gelbart and Jacquet $([\mathrm{GJ}])$, there exists an isobaric automorphic representation $\operatorname{Ad}(\pi)$ of $G L\left(3, \mathbb{A}_{F}\right)$ such that

$$
A_{v}\left(\operatorname{Ad}^{2}(\pi)\right)=\operatorname{sym}^{2}\left(A_{v}(\pi)\right) \otimes \omega^{-1}\left(\varpi_{v}\right)
$$

at every unramified place $v$. Write $A_{v}(\pi)=\left[\alpha_{v}, \beta_{v}\right]$. Then

$$
a_{v}(\operatorname{Ad}(\pi))=\alpha_{v} / \beta_{v}+\beta_{v} / \alpha_{v}+1 .
$$


Claim 4.5. Let $v$ be a finite place where $\pi_{v}$ is unramified. Then we have

$$
a_{v}(\operatorname{Ad}(\pi))+1=a_{v}^{2} \omega\left(\varpi_{v}\right)^{-1}=\left|a_{v}\right|^{2} .
$$

Moreover, if $\left|a_{v}\right| \geq 2$, then $\alpha_{v} / \beta_{v}$ is a positive real number.

Indeed, since $\pi_{v}$ is unitary, we have $\bar{\pi}_{v} \simeq \pi_{v}^{\vee} \simeq \pi_{v} \otimes \omega^{-1}$, and the identity (4.6) results from the decomposition

$$
A_{v}\left(\operatorname{Ad}\left(\pi_{v}\right) \boxplus 1\right)=A_{v}\left(\pi_{v} \times \bar{\pi}_{v}\right)=A_{v}\left(\pi_{v}\right) \otimes A_{v}\left(\pi_{v}\right) \otimes \omega\left(\varpi_{v}\right)^{-1} .
$$

The second assertion then follows easily by applying (4.4). Hence the claim.

If $\pi$ is associated to an idele class character of a quadratic extension of $F$, then one knows that $\pi$ satisfies the Ramanujan conjecture. Hence we may, and we will, assume that we are not in this case, so that, by $[\mathrm{GJ}], \operatorname{Ad}(\pi)$ is cuspidal.

Put

$$
\eta=m[1] \boxplus k \operatorname{Ad}(\pi),
$$

where $m, k$ are non-negative integers to be (suitably) chosen below, and [1] is the trivial representation of $G L\left(1, \mathbb{A}_{F}\right)$. Note that $a_{v}(\eta)=m+k a_{v}(\pi)$.

Lemma 4.8. Let $v$ be a prime in $T(\pi, 2)$. Then $v$ lies in $T(\eta, m+3 k)$.

Proof. Let $v$ belong to $T(\pi, 2)$. Then, by Claim 4.5 ,

$$
\left|a_{v}(\operatorname{Ad}(\pi))+1\right| \geq 4 \text {. }
$$

Since by that same claim, $a_{v}(\operatorname{Ad}(\pi))$ is a positive real number (as $\left.\left|a_{v}(\pi)\right| \geq 2\right)$, it must in fact be $\geq 3$. The Lemma now follows, since $a_{v}(\eta)=m+k a_{v}(\operatorname{Ad}(\pi))$ by (4.7).

Combining this lemma with (4.4), we get

$$
\bar{\delta}(T(\pi, 2)) \leq \frac{m^{2}+k^{2}}{(m+3 k)^{2}} .
$$

This holds for every choice of (non-negative) pairs $(m, k)$. To obtain the best possible bound, we need to minimize the right hand side. It may be verified that the minimum occurs when $k=3 m$, yielding

$$
\bar{\delta}(T(\pi, 2)) \leq 1 / 10 .
$$

This completes the proof of Theorem A.

Remark 4.10. When $n=2, S(\pi)$ is precisely the set of primes $v$ where the Ramanujan conjecture holds. To see this, let $v \in S(\pi)$, and write $A_{v}$ as $\left[\alpha_{v}, \beta_{v}\right]$. The unitarity of $\pi_{v}$ gives the equality of sets $\left\{\bar{\alpha}_{v}, \bar{\beta}_{v}\right\}=\left\{\alpha_{v}^{-1}, \beta_{v}^{-1}\right\}$. Suppose either $\alpha_{v}$ or $\beta_{v}$ does not have absolute value 1 . Then we must have $\beta_{v}=\bar{\alpha}_{v}^{-1}$. Now, since $\left|a_{v}\right| \leq 2$ by the definition of $S(\pi)$, the identities (4.4) and (4.6) imply that $\left|\alpha_{v}\right|^{2}+\left|\alpha_{v}\right|^{-2} \leq 2$. It is then immediate that $\left|\alpha_{v}\right|$, and hence $\left|\beta_{v}\right|$, is 1 , giving the necesary contradiction. 


\section{Proof of Theorem B}

Let $\pi$ be a unitary, cuspidal, self-dual automorphic representation of $G L\left(n, \mathbb{A}_{F}\right), n \geq 2$, such that the inverse roots $\alpha_{j, v}$ lie in a fixed disk in $\mathbb{C}$, for all $v$ outside a finite set $X$ of places containing the archimedean and ramified places (for $\pi$ ). Let $\mathcal{L}^{*}$ denote the subgroup of $\mathcal{D}^{*}$ generated by the following $L$-series: (i) $L^{X}(s, \pi)$, (ii) $L^{X}(s, \pi \times \pi)$, and (when $n=2$ ) (iii) $L^{X}\left(s, \pi, \operatorname{sym}^{k}\right)$, $k \leq 5$. Then, by the results of Jacquet, Shalika and Piatetski-Shapiro ([JPSS], [JS]) for (i), (ii), and Shahidi ([Sh1,2]]) for (ii), (iii), it is immediate that every element $L(s)$ of $\mathcal{L}^{*}$ satisfies $(\mathrm{H} 1)$, in fact with $\underline{\lambda}(L)=\bar{\lambda}(L)$; call it $\lambda(L)$. It also satisfies $\left(H_{2}\right)$ by our assumption on the $\left|\alpha_{j, v}\right|$. Note that, by the self-duality of $\pi$, the coefficients of all these $L$-series are real.

Let $m, k \in \mathbb{Z}$, with $k \neq 0$. Put

$$
L(s)=L^{X}(s, \pi)^{m} L^{X}(s, \pi \times \pi)^{k} \in \mathcal{L}^{*} .
$$

Then $\lambda(L)=k$, and $a_{v}(L)=m a_{v}(\pi)+k a_{v}(\pi)^{2}$. Putting $r=m / k$ and applying Lemma 1.3 , we get the existence of sets $S_{j}, j=1,2$, of primes with $\underline{\delta}\left(S_{1}\right), \bar{\delta}\left(S_{2}\right)$ positive, such that

$$
a_{v}(\pi)^{2}+r a_{v}(\pi)<1+\epsilon, \quad \forall P \in S_{1},
$$

and

$$
a_{v}(\pi)^{2}+r a_{v}(\pi)>1-\epsilon, \quad \forall P \in S_{2} .
$$

Part (b) of the Theorem B follows easily.

It remains to prove part (a). From now on, let $n=2$. We may, and we will, assume that $\pi$ is not associated to an idele class character of a quadratic extension of $F$. Let us now apply the assertion of part (b) to $\operatorname{Ad}(\pi)$, which is a self-dual, cuspidal automorphic representation of $G L\left(3, \mathbb{A}_{F}\right)$. Using (4.6), we then get, for all $\epsilon^{\prime}>0$, and for every $r \in \mathbb{Q}$, a set $S\left(=S_{2}\right)$ of primes of positive upper density such that

$$
\left|a_{v}(\pi)\right|^{2} \notin\left[1-\left(r+\sqrt{r^{2}+4}\right) / 2+\epsilon^{\prime}, 1+\left(-r+\sqrt{r^{2}+4}\right) / 2-\epsilon^{\prime}\right], \quad \forall v \in S .
$$

If we choose $r$ to be greater than $3 \epsilon^{\prime}$, then $1-\left(r+\sqrt{r^{2}+4}\right) / 2+\epsilon^{\prime}$ is negative, and thus (5.3) simplifies as

$$
\left|a_{v}(\pi)\right|^{2}>1+\left(-r+\sqrt{r^{2}+4}\right) / 2-\epsilon^{\prime}, \quad \forall v \in S .
$$

Now for any $\epsilon>0$, we can choose $\epsilon^{\prime}$ and rational number $r>3 \epsilon^{\prime}$ such that $\left(-r+\sqrt{r^{2}+4}\right) / 2-\epsilon^{\prime}>1-\epsilon$. Thus part (a) of Theorem B follows.

Remark 5.5. Note that the assertion concerning cusp forms $\pi$ on $G L(2) / F$ (in Theorem B) is proved in effect by consideration of the $L$-series

$$
L^{X}\left(s, \pi, \operatorname{sym}^{4}\right)^{a} L^{X}\left(s, \pi, \operatorname{sym}^{2}\right)^{b} \zeta_{F}^{X}(s)^{c},
$$


for suitable integers $a, b, c$, when $\omega_{\pi}=1$. Instead of exploiting the automorphy of $\operatorname{sym}^{2}(\pi)([\mathrm{GJ}])$, we can also directly use the results of Shahidi ([Sh 1,2]) and arrive at the same conclusion.

We end this section clarifying certain points at the suggestion of Shahidi, but there will be no new results from here on. Using the method above, one can also recover some results of Serre in the appendix of [Sh2]. For example, if we let $r=-8 / 3$ and take $S_{2}$ as in (5.2) applied to $\operatorname{Ad}(\pi)$, we will get

$$
\left|a_{v}(\pi)\right|>\sqrt{\frac{2}{3}}-\epsilon, \quad \forall v \in S_{2},
$$

which gives, as Serre remarks, the best possible lower bound with current knowledge. Moreover, we can prove (in the same vein) the following result, which is immediate from the discussion in [Sh2], appendix:

Proposition 5.6. Let $\pi$ be a non-monomial, self-dual, cuspidal automorphic representation of $G L\left(2, \mathbb{A}_{F}\right)$ with coefficients $\left\{a_{v}\right\}$ such that, for a large enough finite set $X$ of primes in $F$, the $L$-series $L^{X}\left(s, \pi, \mathrm{sym}^{5}\right)$ is invertible at $s=1$. Fix $\epsilon>0$. Then there are sets $S^{+}$and $S^{-}$of primes of positive lower (Dirichlet) density such that

$$
a_{v}>\sqrt{2}-\epsilon, \quad \forall v \in S^{+}, \text {and } a_{v}<-\sqrt{2}+\epsilon, \quad \forall v \in S^{-} .
$$

Let us note that Shahidi has proved the meromorphic continuation of $L^{X}\left(s, \pi, \operatorname{sym}^{5}\right)$ and has shown that, at $s=1$, it is either invertible or has a simple pole or zero.

We will now show how to prove the first part of Prop. 5.6. The second part follows by an obvious variant of the argument, and is left to the reader. The basic idea is to find an $L(s)$ in the group $\mathcal{G}^{*} \leq \mathcal{D}^{*}$ generated by $\left\{L^{X}\left(s, \pi, \operatorname{sym}^{j}\right) \mid 1 \leq\right.$ $j \leq 5\}$ such that the coefficients $b_{v}$ of $L(s)$ are given by the values at $a_{v}$ of an integral quintic polynomial $f(x)$ with positive leading term, having a unique real root $b$ arbitrarily close to $\sqrt{2}$. Then by the assumption on $L^{X}\left(s, \pi, \operatorname{sym}^{5}\right)$ and the known results on the lower symmetric power $L$-functions (cf. [Sh 1,2]), $L(s)$ is well defined and invertible at $s=1$, and the assertion follows by applying Lemma 1.3.

It suffices to find a monic polynomial $f(x)$ with rational coefficients satisfying the conditions above. For each $j \geq 1$, let $T_{j}$ denote the polynomial (in $x$ ) giving $a_{p}\left(\pi, \operatorname{sym}^{j}\right)$, so that $T_{1}=x, T_{2}=x^{2}-1, T_{3}=x^{3}-2 x, T_{4}=x^{4}-3 x^{2}+1$, and $T_{5}=x^{5}-4 x^{3}+3 x$. Put

$$
f(x)=T_{5}+\left(z+u^{2}+4\right) T_{3}+\left(2\left(z+u^{2}\right)-5-u^{2} z\right) T_{1}+y\left(T_{4}+\left(u^{2}+1\right) T_{2}\right),
$$

where $y, z, u$ are non-zero rational numbers to be chosen. (It is clear that an integral multiple of $f(x)$ gives the coefficients of some $L(s) \in \mathcal{G}^{*}$, for any choice of $(y, z, u)$.) Then we have

$$
f(x)=\left(x^{2}+u^{2}\right)\left(x^{3}+z x+y\left(x^{2}-2\right)\right) .
$$


Choose non-zero rational numbers $a, t$ and set

$$
y=2 a-b, \quad \text { and } \quad z=t^{2}+a^{2}-2 a b,
$$

where $b=4 a /\left(2+a^{2}+t^{2}\right)$. Then we get

$$
f(x)=\left(x^{2}+u^{2}\right)\left((x+a)^{2}+t^{2}\right)(x-b) .
$$

We claim that the unique real root $b$ can be made to lie in $(\sqrt{2}-\epsilon, \sqrt{2})$, for any $\epsilon$, by a suitable choice of $a, t$. Indeed, the (unallowable) limiting case $(a, t)(\sqrt{2}, 0)$ corresponds to $b=\sqrt{2}$ (and $f(x)=\left(x^{2}+u^{2}\right)(x+\sqrt{2})^{2}(x-\sqrt{2})$ ). This shows that by choosing $(a, t)$ to be close, but not equal, to $(\sqrt{2}, 0)$, we can make $b$ arbitrarily close to $\sqrt{2}$ (from below). For example, put $a=1.414$ and $t=0.01$. Then

$$
b=1.414178186451492888103901091 \ldots .
$$

\section{Acknowledgements}

I would like to thank Freydoon Shahidi for his interest in this project since its inception and for helpful conversations, David Roberts for a useful discussion of Sato-Tate measures, Dipendra Prasad for looking over the argument and finding bugs, and J.-P. Serre for his helpful comments on an earlier version. Thanks are also due to the referee for good suggestions. One can ask for analogs of our results for natural density and also ask for effective versions and omega results. We hope to address these questions elsewhere.

\section{References}

[BL] J.-L. Brylinski and J.-P. Labesse, Cohomologie d'intersection et fonctions L de certaines variétés de Shimura, Ann. Scient. Ecole Norm. Sup. (4) 17 (1984), 361-412.

[BR] D. Blasius and J. Rogawski, Motives for Hilbert modular forms, Invent. Math. 114 (1993), 55-87.

[BDHI] D. Bump, W. Duke, J. Hoffstein, and H. Iwaniec, An estimate for the Hecke eigenvalues of Maass forms, Internat. Math. Res. Notes 4 (1992), 75-81.

[Ca] H. Carayol, Sur les représentations $\ell$-adiques associées aux formes modulaires de Hilbert, Ann. Scient. Ecole Norm. Sup. (4) 19 (1986), 409-468.

[C] L. Clozel, Représentations galoisiennes associées aux représentations automorphes autoduales de $G L(n)$, Inst. Hautes Études Sci. Publ. Math. 73 (1991), 97-145.

[D] P. Deligne, Formes modulaires et représentations $\ell$-adiques, Sém. Bourbaki 1968/69, no. 355, Lecture Notes in Math. 179, Springer, New York.

[DS] P. Deligne and J.-P. Serre, Formes modulaires de poids 1, Ann. Scient. Ecole Norm. Sup. (4) 7 (1974), 507-530.

[GJ] S. Gelbart and H. Jacquet, A relation between automorphic representations of GL(2) and $G L(3)$, Ann. Scient. Ecole Norm. Sup. (4) 11 (1979), 471-542.

[HR] J. Hoffstein and D. Ramakrishnan, Siegel zeros and cusp forms, Intenat. Math. Res. Notes 6 (1995), 279-308.

[JS] H. Jacquet and J. Shalika, Euler products and the classification of automorphic forms I $\&$ II, Amer. J Math. 103 (1981), 499-558 \& 777-815.

[JPSS] H. Jacquet, I. Piatetski-Shapiro, and J. Shalika, Rankin-Selberg convolutions, Amer. J Math. 105 (1983), 367-464. 
[Lf] L. Lafforgue, Sur la conjecture de Ramanujan-Petersson pour les corps de fonctions. I: étude géométrique \& II: étude spectrale, C. R. Acad. Sci. Paris Ser. I 322 (1996), 605-608 \& 707-710.

[L1] R. P.Langlands, Problems in the theory of Automorphic Forms, in "Lectures in Modern Analysis and Applications", Springer Lecture Notes 170 (1970), 18-61.

[L2] _ Automorphic representations, Shimura varieties and motives, Ein Märchen, Proc. Symp. Pure Math. 33, ed. by A. Borel and W. Casselman, part 2, Amer. Math. Soc. (1979), 205-246.

[LRS] W. Luo, Z. Rudnick, and P. Sarnak, On the generalized Ramanujan conjecture for $G L(n)$, preprint (1997).

[Mu] M. R. Murty, Oscillations of Fourier coefficients of modular forms, Mathematische Annalen 262 (1983), 431-446.

[Pic] Zeta functions of Picard modular surfaces, Centre de Recherches Mathématiques Publications, ed. by R. P. Langlands and D. Ramakrishnan, Montréal (1992).

[Ra] D. Ramakrishnan, A refinement of the strong multiplicity one theorem for GL(2), Appendix to [T2], Invent. Math. 116 (1994), 645-649.

[Sa] P. Sarnak, Statistical properties of eigenvalues of Hecke operators, in: Analytic Number theory and Diophantine problems (Stillwater, OK), 321-331, ed. by A. C. Adolphson, J. B. Conrey, A. Ghosh and R. I. Yager, Progr. Math. 70, Boston (1987).

[Se] J.-P. Serre, Propriétés conjecturales des groupes de Galois motiviques et des représentations $\ell$-adiques, in "Motives", part 1, Proc. Symp. Pure Math. 55, ed. by U. Jannsen, S. Kleiman and J.-P. Serre (1991), 377-400.

[Sh1] F. Shahidi, On the Ramanujan conjecture and the finiteness of poles for certain $L$ functions, Ann. of Math. (2) 127 (1988), 547-584.

[Sh2] , Symmetric power L-functions for $G L(2)$, in: Elliptic curves and related topics, Centre de Recherches Mathématiques Proceedings \& Lecture Notes 4, ed. by H. Kisilevsky and M. R. Murty, Montréal (1994).

[T1] R. Taylor, On Galois representations associated to Hilbert modular forms, Invent. Math. 98 (1989), 265-280.

[T2] — - $\_$-adic representations associated to modular forms over imaginary quadratic fields II, Invent. Math. 116 (1994), 619-643.

[W] A. Wiles, On ordinary $\lambda$-adic representations associated to modular forms, Invent. Math. 94 (1988), 529-573.

Dept. of Math. 253-37, California Institute of Technology, Pasadena, CA 91125

E-mail address: dinakar@cco.caltech.edu 